\title{
Many-body Interactions in Magnetic Films and Nanostructures Final Report
}

\section{Research Accomplishments}

\section{a. Electronic structure of stepped surfaces}

We characterized the electronic structure of clean stepped surfaces. This is simple step along the path to measuring the electronic and magnetic structure of magnetic nanowires on the same surfaces, but also produced interesting results in its own right. Fig. 1 shows a schematic of the W(3 20$)$ surface, which consists of monatomic steps of $[-1,1,0]$ orientation and five-atom (2.5 bcc unit cell) terraces of [110] orientation.

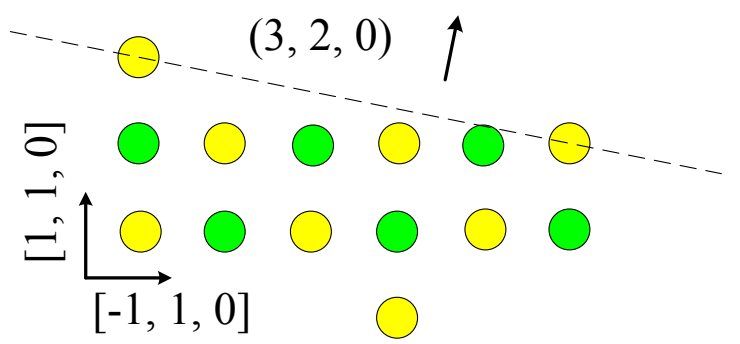
Fig. 1: Schematic side view of the $\mathrm{W}(320)$ surface which nominally has monatomic steps alternating with 5 -atom (2.5 bcc unit cell) terraces. The yellow atoms are lattice atoms, the green atoms are body-center atoms.

Band structures and Fermi contours of the clean W(3 20$)$ surface are shown in Figure 2. An interesting feature in these results is the significant splitting of the $d$ band near the $\bar{S}$ point of the (110) SBZ. This splitting can be observed at different photon energies and different binding energies, particularly below the Fermi level (left panel).
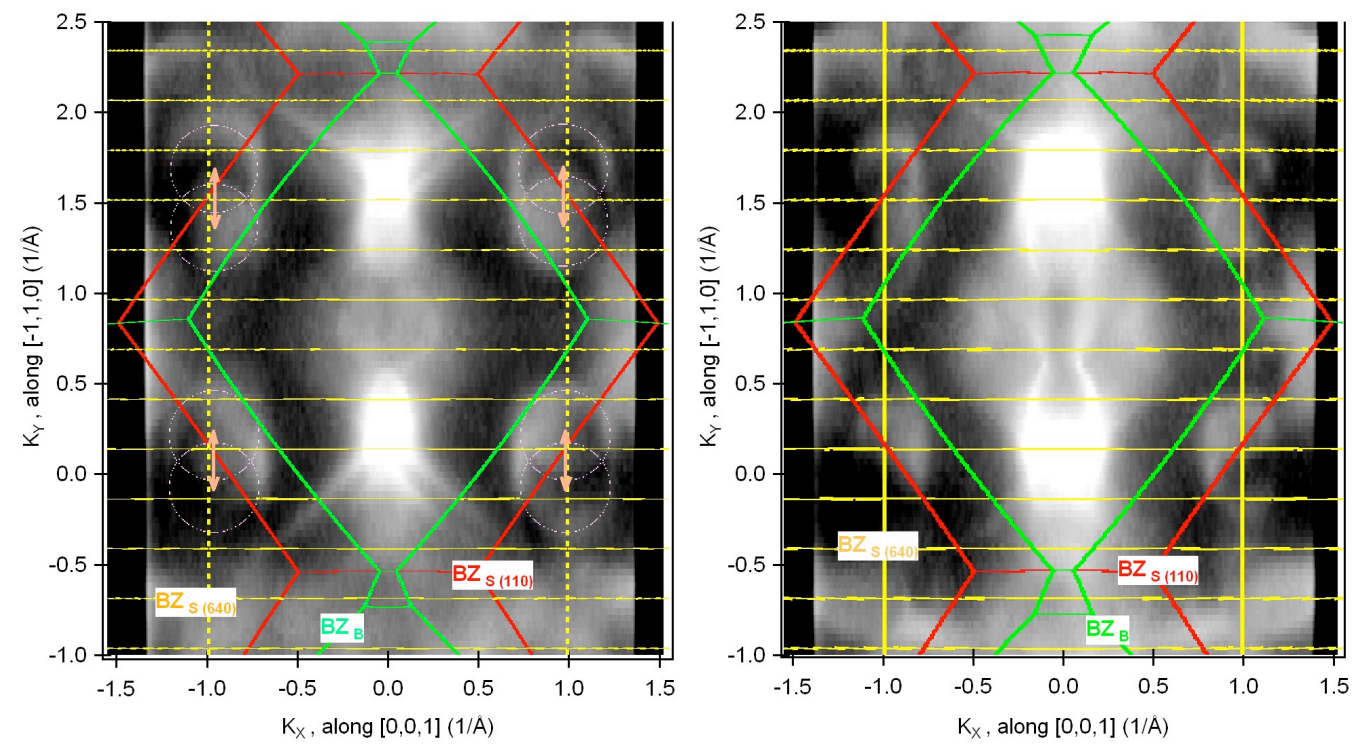

Fig. 2: Iso-energy band contours of clean the W(3 20$)$ surface. Left, binding energy of $0.5 \mathrm{eV}$; right, the Fermi energy contour. Brillouin zone boundaries of $\mathrm{W}$ bulk $\left(\mathrm{BZ} Z_{\mathrm{B}}\right), \mathrm{W}(110)$ surface geometry $\left(\mathrm{BZ} \mathrm{S}_{\mathrm{S}(10)}\right)$ and $\mathrm{W}(6 \quad 40)$ surface geometry $(\mathrm{BZ} \mathrm{S} \mathrm{(640))})$ are drawn and labeled. In the left panel, the surface $\mathrm{d}$ bands at each $\bar{S}$ points on (110) surface Brillouin Zone are labeled with dotted circles. The translation vector between the centers of the two circular orbits at $\bar{S}$ are labeled with orange arrows and match the W(6 40 ) SZB very well. 
Both 2D and 1D phenomena can happen on stepped surfaces. The key factor that determines the behavior of surface electron state is the width of terrace. For larger terrace width, 1D quantum wells $(\mathrm{QW})$ can form on the terrace and give the surface states fine energy splitting. The standing wave produced by QW defines the $1 \mathrm{D}$ behavior of electron, and coupling between terraces represents a 2D deviation from that behavior. With narrower terraces, QW's may not be observed, either because they lie above the Fermi level or because the 2D behavior dominates. In either case, band back-folding can be observed and a band momentum splitting being equal to a reciprocal translation vector $\mathbf{G}_{\mathrm{T}}$ determined by the expected terrace width,

$$
\mathbf{G}_{\mathbf{T}}=2 \pi /(\text { Step period })
$$

can be obtained. The observed K splitting between two $\bar{S}$ d bands, as shown in Fig. 2 on the left, are around $0.28 \AA^{-1}$, which contradicts the expected W(3 20$)$ step periodicity but fits well with a doubled W(6 40$)$ periodicity. Similarly, by analyzing the related k-space splitting of the clean W(8 70 ) surface, a doubled (16 14 0) surface periodicity was found.

The source of this period doubling is not presently understood. Possibly the steps are diatomic, in which case the period would naturally be doubled, though this would be at odds with several papers in the literature. Alternatively, the terraces could alternate 4atoms and 4-atoms in width, due to a longe-range step-step interaction or a propensity to have an even number of bcc unit cells in a terrace. Finally, this doubling could reflect an electronic or magnetic instability, the latter possibly due to a modified Rashba splitting of the surface states near the steps.

\section{b. Localization in graphene caused by H-atom adsorption}

Magnetism in graphite has been a hot issue for some time, and predictions exist that various species bonded to graphene, even $\mathrm{H}$ atoms, are magnetic. Our experiment involved cracking hydrogen molecules to produce $\mathrm{H}$-atoms, which in turn bond at random locations on a graphene film grown on a $\mathrm{SiC}$ substrate. The electronic structure, in particular the coherence of the bands in the vicinity of the Fermi level were then probed using photoemission as a function of hydrogen coverage/doping.

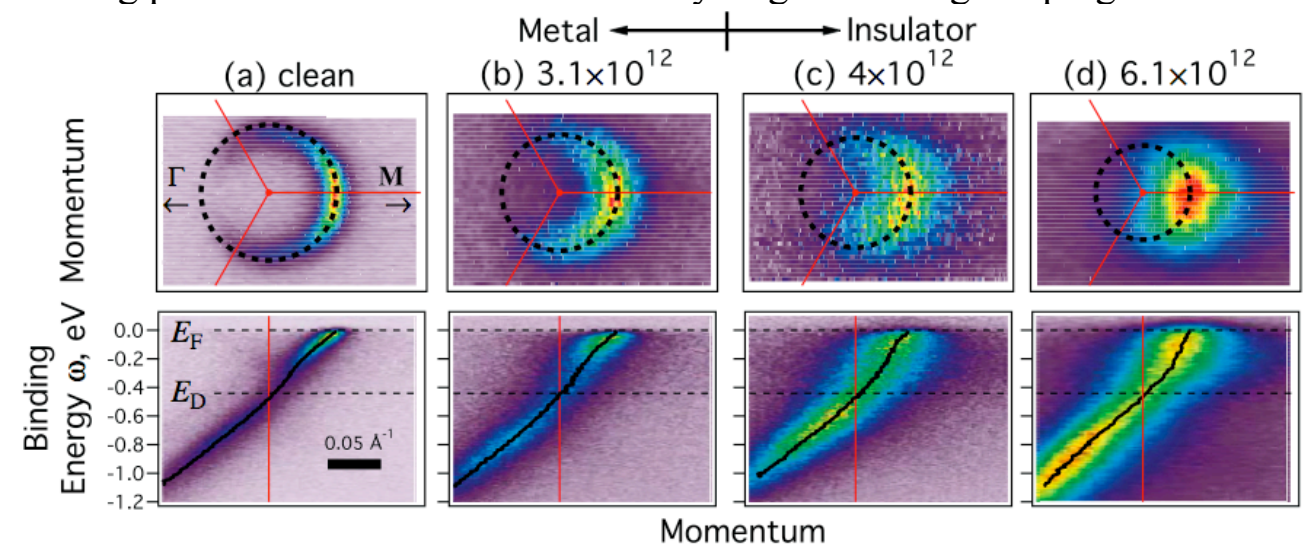

Fig. 3: Fermi surfaces (upper) and associated band structure cuts (lower) through the graphene K point for (a) clean, and (b)-(d) as a function of $\mathrm{n}_{\mathrm{H}}$ indicated in $\mathrm{H}$ atoms per $\mathrm{cm}^{2}$. The dashed lines schematically show the Fermi surface contours. The solid lines in the lower row are the peak position determined by fits to the momentum distribution curves. The data were acquired with a slow flux of $\mathrm{H}$ atoms to compensate for photodesorption of $\mathrm{H}$ atoms. 
The key photoemission results are shown in Fig. 3, which show the band dispersions near the $\mathrm{K}$ point of the 2D Brillouin zone as a function of hydrogen coverage/doping. The lower panel shows how the band becomes very incoherent for even fairly low coverage. Simultaneous measurement of the photovoltage caused by the incident $\mathrm{x}$-ray beam allowed the resistivity of the film to be determined and correlated with the band coherence. A distinct transition was detected that we ascribed to Anderson localization, with a minimum metallic resistivity that is close to the Ioffe, Regel, and Mott (IR\&M) criterion.

\section{c. Metal films adsorbed onto graphene.}

The image shown below was collected from a gold film on graphene of indeterminate average thickness adsorbed on graphene and shows bulk gold band 6 near the Fermi level, split by a well-known Rashba/spin-orbit effect, and also forming a plethora of quantum well states corresponding to a film thickness of $\sim 30 \mathrm{~nm}$. The results require this thickness with very high precision, and the film spontaneously forms 'mesas' of this thickness. Efforts to understand the diverse features in this image continue.

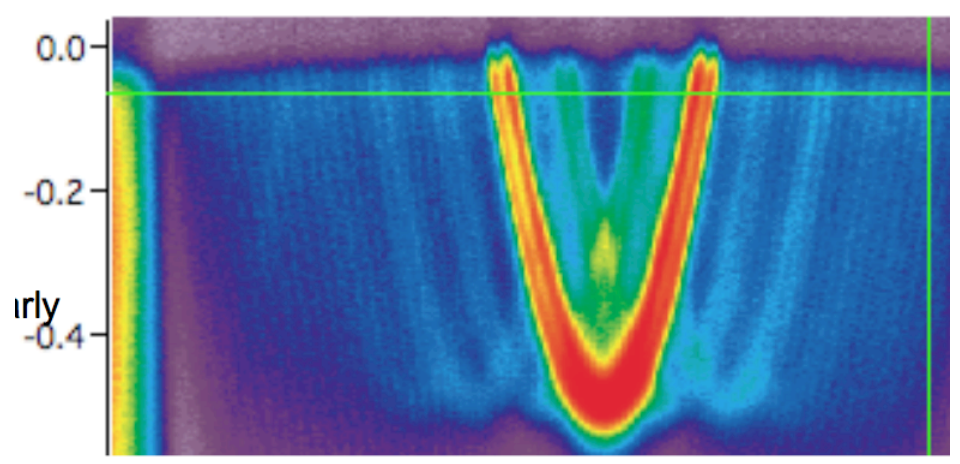

Fig. 4: Dispersion of band 6 of a gold film $\sim 30 \mathrm{~nm}$ thick adsorbed on a grapheme layer supported on a silicon carbide substrate. The main band in res is split by the Rashba splitting, while a multitude of quantum well states are observed at larger momentum.

\section{Papers published supported by this grant}

O. Krupin, G. Bihlmayer, K. Döbrich, J. E. Prieto, K. Starke, S. Gorovikov, S. Blügel, S. Kevan, G. Kaindl, Rashba effect at the surfaces of rare-earth metals and their monoxides, New. J. Phys. 11, 013035 (2009).

Aaron Bostwick, Jessica L. McChesney, Konstantin V. Emtsev, Thomas Seyller, Karsten Horn, Stephen D. Kevan, and Eli Rotenberg, Quasiparticle Transformation during a Metal-Insulator Transition in Graphene, Phys. Rev. Lett. 103, 056404 (2009).

Aaron Bostwick, Taisuke Ohta, Jessica L McChesney, Konstantin V Emtsev, Florian Speck, Thomas Seyller, Karsten Horn, Stephen D Kevan and Eli Rotenberg, The interaction of quasi-particles in graphene with chemical dopants, New. J. Phys. 12, 125014 (2010). 


\section{Personnel}

The project supported the activities of PI S.D. Kevan, including typically 1 or 1.5 months of summer salary.

We collaborate closely with Eli Rotenberg, the first of my ALS postdocs, who took a position at the ALS in the fall of 1996 . He did not receive any direct support from this grant.

The grant also supported full time postdoctoral associate Zhuo Bao, who was involved in all of our experiments at the ALS. He unfortunately arrived very late due to a delay in obtaining a visa, and ended up leaving the program after 1.5 years. This largely explains our reduced publication rate in recent years. 